\title{
The Model of Time and Frequency Resource Scheduling in Downlink LTE
}

\author{
Sergey V. Garkusha
}

\begin{abstract}
We apply the result of the model of time and frequency resource scheduling in downlink LTE development. The given model is directed upon securing the wireless network users being served by the allocation to user equipment required transmission rates. We completed comparative analysis of the offered model with existing methods from the point of view of securing the common productivity of downlink, level of bandwidth balancing, possibility of allocation to users equipment required transmission rate. We showed that the offered model under the highly required conditions to the transmission rate of user equipment in comparison with known methods let increase the bandwidth balancing of downlink for 5-20 \% and 40-100 \% to increase possibility of allocation the required transmission rate to user equipment.
\end{abstract}

Index Terms-LTE, time-frequency resource, resource block, scheduling block, required transmission rate

\section{INTRODUCTION}

$\mathrm{I}_{\mathrm{p}}^{\mathrm{v}}$ LTE developed by 3GPP, one of the effective ways of productivity and quality of service (QoS) increasing is network protocols and mechanisms responsible for available net resources improvement. They are, first of all time resource - OFDM-symbols and frequency resource - subcarrier. OFDM-symbol is period of time during of which amplitude and phase of modulated subcarriers is constant. The task solving about radio resources allocation is based upon requirements to QoS and can be placed on radio resources management (RRM) system, upon scheduler inside the system. In LTE as well as in HSDPA or WiMAX, mechanisms of downlink resources are not defined by the standard, leaving the choice behind the evolved NodeB (eNodeB) equipment producers [1]-[3].

The result of the task solution about allocation of frequency and time resources must be allocation scheduling block (SB) to user equipment (UE) in download of a single frame. Scheduling block is the least structure element, allocated by a single UE and framing two neighboring resources blocks (RB) upon similar subcarriers [4].

In the given research work we analyzed methods of frequency and time allocation, with the algorithm Round Robin Scheduler [3], [5], [6]. Round Robin Scheduler algorithm using supposes equal allocation of frequency and time resource for every UE. In basis realization Round Robin

S.V. Garkusha is with the Poltava University Economics and Trade, Poltava, 36014, Ukraine (e-mail: sv.garkusha@mail.ru).
Scheduler allocates to every UE time interval, under which this UE gets monopoly access to the channel. While this rate of data transmission UE is confined by factual meaning of signal to noise ratio (SNR). We also analyzed methods [5], [6], using algorithm Max C/I Ration, providing frequency and time resource UE with the best SNR meaning, without securing fair allocation the resource between UE. Besides, we analyzed methods of frequency and time allocation, using algorithm of Proportional Fair Scheduling [5]-[7]. The conducted analysis showed that Proportional Fair Scheduling promotes UE which has high SNR meaning, simultaneously providing enough quantity of frequency and time resources UE with worse meaning of SNR.

Analysis showed that the most favorable mechanism for giving access to frequency and time resource LTE would be mechanism, including peculiarities of Round Robin Scheduler and Max C/I Ration algorithms. The choice of algorithm depends on category and load intensity. The right choice of algorithm for giving access is particularly important under high intensity.

At the result of analysis we estimated that using Proportional Fair Scheduling algorithm is directed to apply interactive "best effort" data class to avoid situation when some UE never get access to frequency and time resource. The given class of service $(\mathrm{CoS})$ usage secures data delivery UE as far as possible without data transmission rate guarantee. Improvement the service quality under frequency and time resource scheduling of every UE must be directed upon guaranteed transmission rate with access availability to additional (non-guaranteed) after bandwidth line. However none of analyzed mechanisms is able to secure such CoS.

At the result of conducted analysis we made decision about necessity of frequency and time resource model in downlink LTE scheduling, formulated as allocation SB task for securing guaranteed rate of UE.

\section{MODEL OF RESOURCE BLOCK ALLOCATION IN DOWNLINK}

The offered model is directed for application in wireless networks LTE, using frequency and time channel division. At the model development we consider the fact that the least structure unit of radio resource to be managed at the scheduling task solving SB [1].

LTE suggests three types of resources allocation. The developing model is directed upon resource allocation type 0 , supposing uniting RB in so-called resource blocks groups (RBG), which are allocated for UE. And the quantity of RB, 
enlisted in single $\operatorname{RBG}(p)$, depends on used width of frequency channel. In case if division $\mathrm{RB}$ on parameter $p$ doesn't give integer number, the edging RBG will have size, less than $p[8]$.

To account subframes, allocated at the data travelling in downlink [1], [2], there was given the idea of matrix of configurations of downlink. Matrix is rectangular with number of lines corresponding to quantity of frame configurations $(L)$ and with number of columns corresponding to quantity of subframes $(K)$ in frame i.e.

$$
H=\left\|h_{l, k}\right\|,(l=\overline{0, L-1} ; k=\overline{0, K-1}),
$$

where $h_{l, k}=\left\{\begin{array}{l}1, \text { for } k \text {-th subframe at } l \text { - th configurations } \\ \text { used for data transmission in downlink; } \\ 0, \text { otherwise. }\end{array}\right.$

While scheduling blocks allocation task solving within the scope of the offered model it is necessary to secure the calculation of boolean control variable $\left(x_{k, m}^{n}\right)$ defining the order of allocation scheduling blocks

$$
x_{k, m}^{n}=\left\{\begin{array}{l}
1, \text { if } m-\text { th scheduling block in } k-\text { th } \\
\quad \text { subframe allocated } n-\text { th UE; } \\
0, \text { otherwise }
\end{array}\right.
$$

where $m=\overline{0, M-1} ; k=\overline{0, K-1} ; n=\overline{1, N} ; M$ - number SB formed during single subframe transmission; $N$ - number UE.

At sought date calculation $x_{k, m}^{n}$ it is necessary to complete a number of conditions/constraints:

1) Condition of allocation $m$-th SB of downlink during transmission $k$-th subframe no more that to one UE

$$
\sum_{n=1}^{N} x_{k, m}^{n} \leq 1 \quad(k=\overline{0, K-1} ; m=\overline{0, M-1}) .
$$

2) Condition of allocation UE scheduling block only of downlink:

$$
\frac{\sum_{m=0}^{M-1} \sum_{n=1}^{N} x_{k, m}^{n}}{M} \leq h_{l, k},
$$

where ( $k=\overline{0, K-1} ; l$ - used frame configuration).

3) Condition of allocation for $n$-th UE number of SB, securing necessary rate of transmission in downlink using scheme modulation and coding (MCS):

$$
\begin{aligned}
& \sum_{m=\frac{M}{2}-3}^{\frac{M}{2}+2} R_{S B}^{0} x_{0, m}^{n}+\sum_{m=\frac{M}{2}-3}^{\frac{M}{2}+2} R_{S B}^{5} x_{5, m}^{n}+\sum_{m=0}^{\frac{M}{2}-4} R_{S B}^{0-9} x_{0, m}^{n}+ \\
& +\sum_{m=\frac{M}{2}+3}^{M-1} R_{S B}^{0-9} x_{0, m}^{n}+\sum_{m=0}^{\frac{M}{2}-4} R_{S B}^{0-9} x_{5, m}^{n}+\sum_{m=\frac{M}{2}+3}^{M-1} R_{S B}^{0-9} x_{5, m}^{n}+ \\
& +\sum_{m=0}^{M-1} \sum_{k=0}^{4} R_{S B}^{0-9} h_{l, k} x_{k, m}^{n}+\sum_{m=0}^{M-1} \sum_{k=6}^{K-1} R_{S B}^{0-9} h_{l, k} x_{k, m}^{n} \geq R_{\mathrm{req}}^{n},
\end{aligned}
$$

at $n=\overline{1, N}, l-$ used frame configuration. There are used the next variables in the expression: $R_{S B}^{0}=\left(\left(N_{s y m b}^{R B} N_{S F}^{R B}-\right.\right.$ $\left.\left.N_{P D C C H}-N_{P S S, S S S}-N_{P B C H}\right) K_{s}-N_{p i l o t} N_{S F}^{R B}\right) R_{c}^{n, m} k_{b}^{n, m} / K T_{S F}-$ bandwidth SB [9], formed at zero subframe and transmitting Primary Synchronization Signal (PSS), Secondary Synchronization Signal (SSS) and Physical Broadcast Channel (PBCH), where $N_{P S S, S S S}=2, \quad N_{P B C H}=4 \quad$ OFDM-symbols correspondingly, $N_{s y m b}^{R B}$ - quantity OFDM- symbols, forming one resource block, $N_{S F}^{R B}=2$ - quantity $\mathrm{RB}$, forming at similar subcarriers and allocating UE during single subframe transmitting, $N_{P D C C H}=3$ - quantity OFDM- symbols in each subframe, allocated for transmitting Physical Downlink Control Channel (PDCCH), $N_{\text {pilot }}=4$ - recourse element (RE), allocated for pilot signal transmission in single $\mathrm{RB}, K_{s}$ - number of subcarriers for data transmission in single RB and single $\mathrm{SB}, R_{c}^{n, m}$ - rate of code, used for signal coding $n$-th $\mathrm{UE}$ at subcarriers $m$-th SB, $k_{b}^{n, m}$ - bit loading OFDMsymbol $n$-й UE at subcarriers $m$-th SB, $T_{S F}=1 \mathrm{mc}-$ time of single subframe transmission; $R_{S B}^{5}=\left(\left(N_{s y m b}^{R B} N_{S F}^{R B}-N_{P D C C H}-\right.\right.$ $\left.\left.N_{P S S, S S S}\right) K_{s}-N_{\text {pilot }} N_{S F}^{R B}\right) R_{c}^{n, m} k_{b}^{n, m} / K T_{S F}$ - bandwidth SB [9], formed at fifth subframe and transmitting signal PSS and SSS; $R_{S B}^{0-9}=\left(\left(N_{\text {symb }}^{R B} N_{S F}^{R B}-N_{P D C C H}\right) K_{s}-N_{\text {pilot }} N_{S F}^{R B}\right) R_{c}^{n, m} k_{b}^{n, m} / K T_{S F}$

- bandwidth SB [9], formed on all subframe and containing only PDCCH from service information; $R_{r e q}^{n}-$ required rate of transmission $n$-th UE.

4) Condition of uniting RB and RGB sizes, sutisfacting width of used channel:

$$
\begin{aligned}
& x_{k, m}^{n}=\frac{1}{p} \sum_{z=\left[\left.\frac{m}{p}\right|_{p}\right.}^{\left(\left\lfloor\frac{m}{p}\right\rfloor+1\right) p-1} x_{k, z}^{n}, \\
& \text { at } n=\overline{1, N} ; k=\overline{0, K-1} ; m=\overline{0,\left\lfloor\frac{M}{p}\right\rfloor p-1} \text {; } \\
& x_{k, m}^{n}=\frac{1}{p} \sum_{z=\left[\frac{M}{p}\right]_{p}^{M}}^{M} x_{k, z}^{n}, \\
& \text { at } n=\overline{1, N} ; k=\overline{0, K-1} ; m=\overline{\left\lfloor\frac{M}{p}\right\rfloor p-1, M} \text {, }
\end{aligned}
$$

where $L\rfloor$ is symbol of rounding down to the nearest integer. Condition (7) used for uniting SB of edging RBG, sizes of which can be less $p$.

Calculation of the sought variables (2) in correspondence with conditions/constraints (3)-(7) is expedient to carry out while solving optimized task, providing minimum or maximum before chosen criterion of allocation task quality 
SB. The task of SB allocation can be solved using optimality criterion, directed upon maximizing of common productivity of downlink. So optimal criterion can be:

$$
\begin{aligned}
& \max \sum_{n=1}^{N}\left(\sum_{m=\frac{M}{2}-3}^{\frac{M}{2}+2} R_{S B}^{0} x_{0, m}^{n}+\sum_{m=\frac{M}{2}-3}^{\frac{M}{2}+2} R_{S B}^{5} x_{5, m}^{n}+\sum_{m=0}^{\frac{M}{2}-4} R_{S B}^{0-9} x_{0, m}^{n}+\right. \\
& +\sum_{m=\frac{M}{2}+3}^{M-1} R_{S B}^{0-9} x_{0, m}^{n}+\sum_{m=0}^{\frac{M}{2}-4} R_{S B}^{0-9} x_{5, m}^{n}+\sum_{m=\frac{M}{2}+3}^{M-1} R_{S B}^{0-9} x_{5, m}^{n}+ \\
& \left.+\sum_{m=0}^{M-1} \sum_{k=0}^{4} R_{S B}^{0-9} h_{l, k} x_{k, m}^{n}+\sum_{m=0}^{M-1} \sum_{k=6}^{K-1} R_{S B}^{0-9} h_{l, k} x_{k, m}^{n}\right),
\end{aligned}
$$

taking into account conditions/constraints (3)-(7). Using criterion of optimality (8) together with conditions/constraints (3)-(7) directed upon securing guaranteed transmission rate with access possibility to additional (non-guaranteed) bandwidth in $\mathrm{CoS}$, corresponds to subclass B.

\section{ANALYSIS OF SCHEDULE BLOCKS TASKS SOLVING}

With the aim to analyze resource blocks in downlink scheduling received by using known methods and offered model (2)-(8), let's consider example where for SB allocation in downlink as input data we used the following: quanttity UE - $N=5$; number $\mathrm{SB}$, formed during single time slot transmission $-M=15$; quantity OFDM-symbols, forming single resource block $-N_{s y m b}^{R B}=7$; effectivity meaning MCS signals UE for different resource blocks (Fig. 1); type of channels division - TDD; quantity of subframes in downlink $K=4$ (configuration frame - №1 [1], [2]); quantity SB entering into the composition on single $\mathrm{RBG}-p=2$.

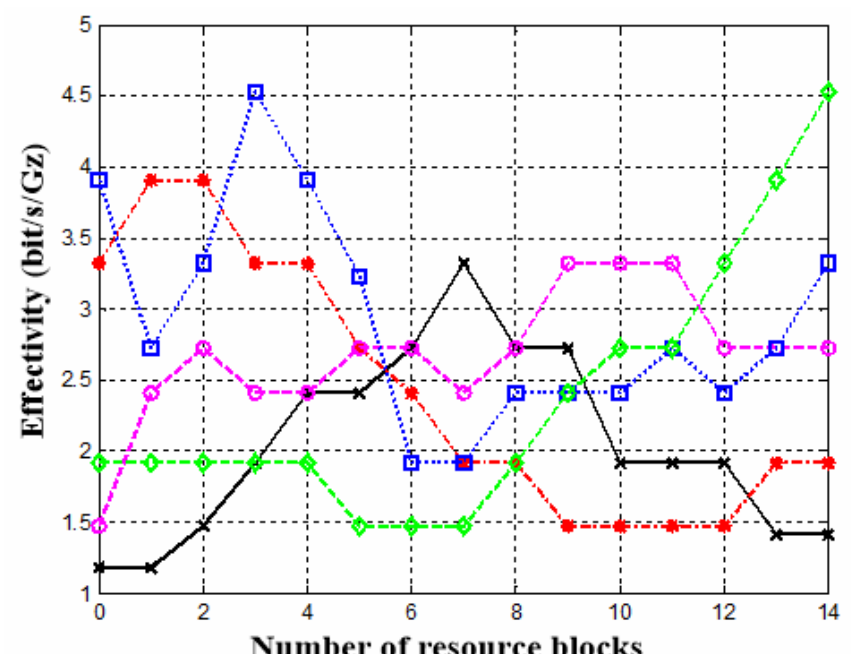

Fig. 1. MCS signals of UE effectivity meaning for different resource blocks, used in the example, where $\rightarrow$ corresponded to the first UE, $-\cdot \cdots--$ the second UE, $--\ominus---$ the third UE, $\cdots \cdot \cdots-$ the fourth UE, $--\diamond---$ the fifth UE

As an example there was received decision of formed during the process optimization problem, using Matlab system
R2012b. While this there was employed program minlpAssign of optimization packet TOMLAB. For example all the UE had same required rates with meaning $R_{\text {req }}^{n}=0 \div 0.26 \mathrm{Mbps}$.

The modelling results showed that common downlink productivity using known methods during all interval of measuring didn't change and made up for Round Robin method - $0.9622 \mathrm{Mbps,}$ Proportional Fair method 1.2377 Mbps, Max C/I Ratio method - 1.4192 Mbps. Common productivity of downlink using offered model (2)-

(8) at the area $R_{\text {req }}^{n}=0 \div 0.15$ Mbps had maximal meaning, corresponding Max C/I Ratio method and made up 1.4192 Mbps. At the interval $R_{\text {req }}^{n}=0,15 \div 0.26$ Mbps common productivity lessened for $3 \%$ to meaning $1.3641 \mathrm{Mbps}$.

At Fig. 2 there are given results of modelling, reflecting dynamics of downlink balancing of bandwidth between UE. Balancing level of bandwidth was defined according to expression [10]

$$
F^{i}=1-\left(\max _{n} R_{n}^{i}-\min _{n} R_{n}^{i}\right) / \sum_{n=1}^{N} R_{n}^{i}
$$

where $R_{n}^{i}$ is rate of transmission, distinguished $n$-th UE at $i$-th interval of measuring, $n=\overline{1, N}$.

The result of modelling (Fig. 2) showed that balancing level of downlink bandwidth using known methods during all the interval of measuring did not change and made up for Round Robin method - 0.9421, Proportional Fair method - 0.9163, Max C/I Ratio method - 0.8214. Balancing level of downlink bandwidth using offered model (2)-(8) at the area $R_{\text {req }}^{n}=0 \div 0.15 \mathrm{Mbps}$ had minimal meaning corresponding to Max C/I Ratio method and made up 0.8214. At interval $R_{\text {req }}^{n}=0.15 \div 0.26$ Mbps balancing level of bandwidth increased to 0.9859 .

At Fig. 3 there are results of requirement fulfilment probability calculation of transmission rate allocated to all UE. Requirement fulfilment probability of transmission rate at $i$ th interval of measurement was defined according to expression

$$
P^{i}=\sum_{n=1}^{N} Q_{n}^{i} / N
$$

where $\sum_{n=1}^{N} Q_{n}^{i}-\mathrm{UE}$ quantity, which distinguished required transmission rate at $i$-th measurement interval, i.e. $Q_{n}^{i}=\left\{\begin{array}{l}0, \text { if } R_{n}^{i}<R_{\mathrm{req}}^{i} \\ 1, \text { if } R_{n}^{i} \geq R_{\mathrm{req}}^{i}\end{array}\right.$

As the modelling results showed at measuring the required transmission rate from $0.15 \mathrm{Mbps}$ and higher all the considered and known methods do not secure required transmission rate meaning for all UE. Fulfillment of the transmission rate requirement by known models is secured only at high meanings $R_{\text {req }}^{n}$ when $R_{\text {req }}^{n}=0 \div 0.15$ Mbps. Using the offered model (2)-(8) secures allocation required transmission rate $\mathrm{UE}$ at whole measurement interval 
$R_{\text {req }}^{n}=0 \div 0.26$ Mbps.

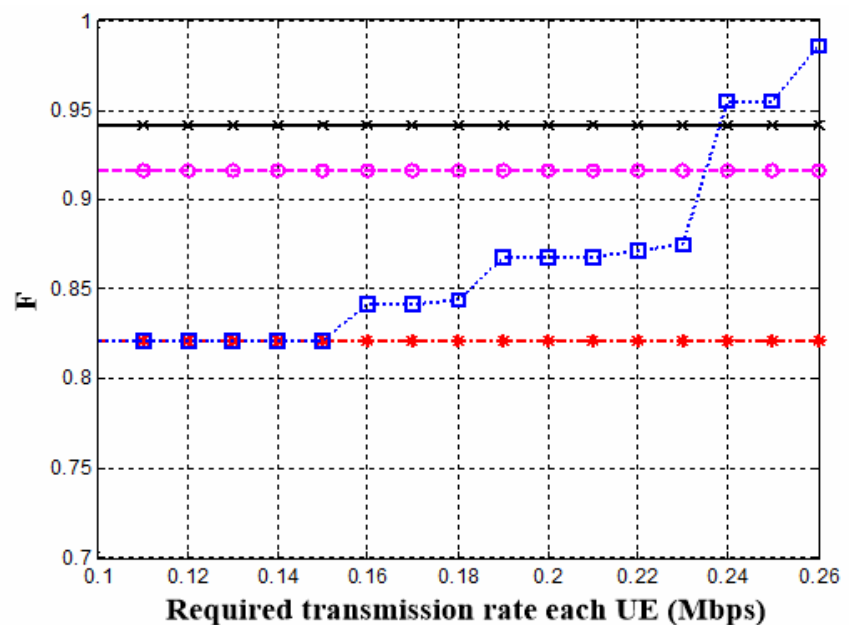

Fig. 2. Balancing level of downlink bandwidth distinguishing UE required transmission rate probability, where $\longrightarrow$ - corresponds Round Robin method, ----Max C/I Ratio method, -- --- - Proportional Fair method, $\cdots \cdot \cdots$ - supposed model

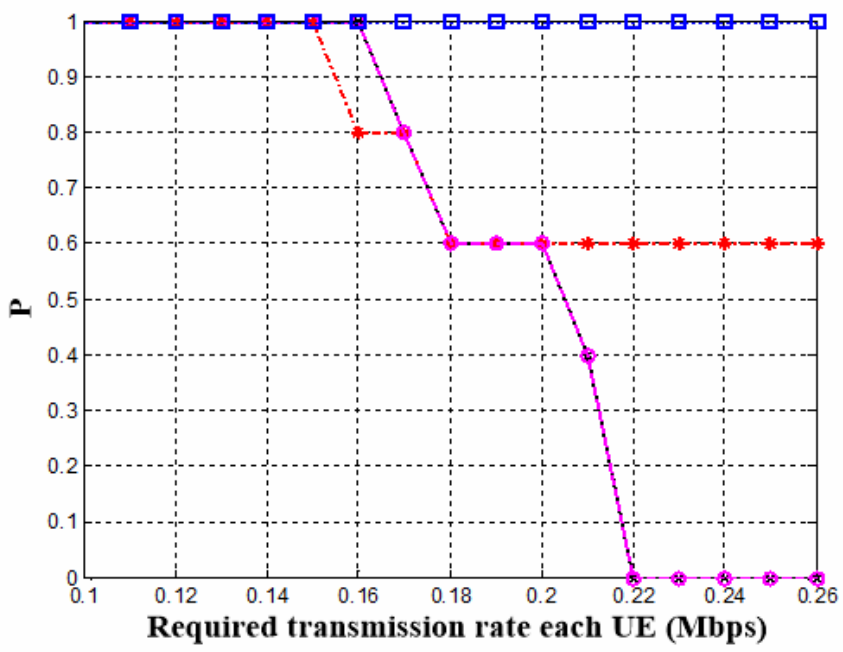

Fig. 3. Distinguishing UE required transmission rate probability, where * corresponds Round Robin method, --*-- -Max C/I Ratio method, -- -- - Proportional Fair method, $\cdots \cdot \square \cdots-$ supposed model

\section{CONCLUSION}

We determined that one of the wireless network basic tasks, functioning with LTE is task of required service quality securing that includes necessity of UE required downlink transmission rate allocation. Securing required LTE transmission rate can be reached by SB allocation in downlink. According to this we analyzed existing mechanisms of SB allocation between UE in downlink of wireless network, functioning with LTE.

On the basis of detected drawbacks of known decisions we suggest the model which is represented by a range of linear and non-linear conditions/constraints. Novelty of model is in formulating the task of SB allocation as task of redistribution available bandwidth of downlink LTE for data transmission to UE taking into account their territorial remoteness (type of modulation and coding system).
Comparative analysis showed that under conditions of high requirements to UE transmission rate, using model (2)-(8) in comparison with known methods lets increase balancing level of downlink bandwidth to 5-20\% (Fig. 2), to increase probability of allocation to UE required transmission rate to $40-100 \%$ (Fig. 3). While this the productivity of downlink under conditions of high requirements to transmission rate $3 \%$ less of productivity received from Max C/I Ratio method and $10-42 \%$ more of productivity using Round Robin and Proportional Fair methods.

The usage of the offered model is directed upon securing each UE guaranteed transmission rate in downlink with access possibility to additional (non-guaranteed) bandwidth. In service classes it corresponds subclass B CoS, when the other methods do not guaranteed required transmission rate what in service classes CoS corresponds to subclass A.

\section{REFERENCES}

[1] 3GPP TS 36.211. 3rd Generation Partnership Project; Technical Specification Group Radio Access Network; Evolved Universal Terrestrial Radio Access (E-UTRA); Physical Channels and Modulation (Release 11). - Valbonne, France: Sophia Antipolis, 2012. - 108 p.

[2] Ghosh A., J. Zhang, R. Muhamed, Andrews J. Cr. Fundamentals of LTE. - Prentice Hall, USA, 2010. - 464 p.

[3] Iosif O., Banica I. On the Analysis of Packet Scheduling in Downlink 3GPP LTE System // The Fourth International Conference on Communication Theory, Reliability, and Quality of Service (CTRQ 2011), pp. 99-102, 2011

[4] Dahlman E., Parkvall S., Skold J., Beming P. $3 G$ Evolution - HSPA and LTE for Mobile Broadband. - Academic Press, 2008. - $608 \mathrm{p}$

[5] Kawser M. T., Farid H. M. A. B., Hasin A. R., Sadik A. M. J., Razu I. K. Performance Comparison between Round Robin and Proportional Fair Scheduling Methods for LTE // International Journal of Information and Electronics Engineering, Vol. 2, No. 5, pp. 678-681, 2012.

[6] Galaviz G., Covarrubias D. H., Andrade A. G., Villarreal S. A resource block organization strategy for scheduling in carrier aggregated systems // EURASIP Journal on Wireless Communications and Networking, pp. 107-124, 2012.

[7] Girici T., Zhu C., Agre J. R., Ephremides A. Proportional Fair Scheduling Algorithm in OFDMA-BasedWireless Systems with QoS Constraints // Journal of communications and networks, Vol. 12, No. 1, pp. $30-42,2010$

[8] 3GPP TS 36.213. 3rd Generation Partnership Project; Technical Specification Group Radio Access Network; Evolved Universal Terrestrial Radio Access (E-UTRA); Physical layer procedures (Release 11). - Valbonne, France: Sophia Antipolis, 2012. - 104 p.

[9] Yermolayev V.T., Flaxman A.G. Teoreticheskie osnovy obrabotki signalov $v$ sistemah mobil'noj radiosvjazi. Nizhnij Novgorod: NGU im. I.N. Lobachevskogo, 2010. - $107 \mathrm{c}$.

[10] Adibah M. R. H., Kumbesan S., Riyaj B., Leijia W. Modeling and simulation of packet scheduling in the downlink long term evolution system // Proceedings of 15th Asia-Pacific Conference on Communications (APCC 2009), pp. 68-71, 2009.

Sergey V. Garkusha received the $\mathrm{PhD}$ degree in telecommunication systems and networks in 2009. Currently, he is the asst. prof. in department information activities in economic systems in Poltava University of Economics and Trade and doctoral department of telecommunication systems in Kharkov National University of Radioelectronics. His research interest includes the optimization and quality of service in wireless telecommunications. He is coauthor of one book, author of more than 50 articles, and 3 inventions. 